\title{
COALGEBRA AND COINDUCTION IN DECENTRALIZED SUPERVISORY CONTROL
}

\author{
Jan Komenda ${ }^{1}$ \\ CWI, P.O.Box 94079, 1090 GB Amsterdam, \\ The Netherlands and \\ Mathematical Institute, AS CR, Zizkova 22, CZ - 61662 \\ Brno, Czech Republic \\ E-mail: Jan.Komenda@cwi.nl
}

\begin{abstract}
Coalgebraic methods provide new results and insights for the supervisory control of discrete-event systems (DES). In this paper a coalgebraic framework for the decentralized control of DES is proposed. Coobservability, decomposability, and strong decomposability are described by corresponding relations and compared to each other. Copyright $\mathbb{C} 2003$ IFAC
\end{abstract}

Keywords: Decentralized supervisory control, discrete-event systems, coobservability, coalgebra.

\section{INTRODUCTION}

Discrete-event (dynamical) systems (DES) can be studied using coalgebraic techniques. DES are often represented by automata viewed as a particular algebraic structure. However, they may be also viewed as partial automata, which are coalgebras of a simple functor of the category of sets. Coalgebras are categorial duals of algebras (the corresponding functor operates from a given set rather than to a given set).

This paper presents a formulation of the decentralized control of DES in terms of coalgebra. The basic formalism is the one that has been developed in Rutten (1999) and by the first author in Komenda (2002), i.e. partial automata as models for DES and partial automaton of (partial) languages as the final coalgebra. The main advantage of the use of coalgebra is the naturally algorithmic character of the results, there is a canonical way how to check the properties like decomposability or coobservability by constructing corresponding relations. As an application, the exact relationship

\footnotetext{
1 Partially supported by the Grant 201/03/D77 of GACR
}

between $\mathrm{C} \& \mathrm{P}$ coobservability and decomposability is derived.

The paper is organized as follows. Section 2 recalls the partial automata from Rutten (1999) as the coalgebraic framework for DES represented by automata. In Section 3 after introducing local weak transition structures on partial automata we present an introduction to the decentralized supervisory control. We give an alternative definition of coobservability, which is equivalent to $\mathrm{C} \& \mathrm{P}$ coobservability of and coincides with the original definition of coobservability in the case of two local supervisors. In section 4 relational characterizations of coobservability are given. Section 5 is devoted to the coalgebraic study of decomposability and strong decomposability, that are described by the corresponding relations and compared to coobservability. We show in particular the exact relationship between $\mathrm{C} \& \mathrm{P}$ coobservability and decomposability. 


\section{PARTIAL AUTOMATA}

In this section we recall partial automata as coalgebras with a special emphasis on the final coalgebra of partial automata, i.e. partial automaton of partial languages. Let $A$ be an arbitrary set (usually finite and referred to as the set of inputs or events). The empty string will be denoted by $\varepsilon$. Denote by $1=\{\emptyset\}$ the one element set and by $2=\{0,1\}$ the set of Booleans. A partial automaton is a pair $S=(S,\langle o, t\rangle)$, where $S$ is a set of states, and a pair of functions $\langle o, t\rangle: S \rightarrow 2 \times$ $(1+S)^{A}$, consists of an output function $o: S \rightarrow 2$ and a transition function $S \rightarrow(1+S)^{A}$. The output function $o$ indicates whether a state $s \in S$ is accepting (or terminating) : $o(s)=1$, denoted also by $s \downarrow$, or not: $o(s)=0$, denoted by $s \uparrow$. The transition function $t$ associates to each state $s$ in $S$ a function $t(s): A \rightarrow(1+S)$. The set $1+S$ is the disjoint union of $S$ and 1 . The meaning of the state transition function is that $t(s)(a)=\emptyset$ iff $t(s)(a)$ is undefined, which means that there is no $a$-transition from the state $s \in S . t(s)(a) \in S$ means that $a$-transition from $s$ is possible and we define in this case $t(s)(a)=s_{a}$, which is denoted mostly by $s \stackrel{a}{\rightarrow} s_{a}$. This notation can be extended by induction to arbitrary strings in $A^{*}$. Assuming that $s \stackrel{w}{\rightarrow} s_{w}$ has been defined, define $s \stackrel{w a}{\longrightarrow}$ iff $t\left(s_{w}\right)(a) \in S$, in which case $s_{w a}=t\left(s_{w}\right)(a)$ and $s \stackrel{w a}{\rightarrow} s_{w a}$.

\subsection{Final automaton of partial languages}

Below we define partial automaton of partial languages over an alphabet (input set) $A$, denoted by $\mathcal{L}=\left(\mathcal{L},\left\langle o_{\mathcal{L}}, t_{\mathcal{L}}\right\rangle\right)$. More formally, $\mathcal{L}=\{\Phi$ : $A^{*} \rightarrow(1+2) \mid \operatorname{dom}(\Phi) \neq \emptyset$ is prefix-closed $\}$. To each partial language $\Phi$ a pair $\langle V, W\rangle$ can be assigned: $W=\operatorname{dom}(\Phi)$ and $V=\{w \in$ $\left.A^{*} \mid \Phi(w)=1(\in 2)\right\}$. Conversely, to a pair $\langle V, W\rangle \in \mathcal{L}$, a function $\Phi$ can be assigned : $\Phi(w)=1$ if $w \in V, \Phi(w)=0$ if $w \in W$ and $w \notin V$ and $\Phi(w)$ is undefined if $w \notin W$. Therefore we can write :

$\mathcal{L}=\left\{(V, W) \mid V \subseteq W \subseteq A^{*}, W \neq \emptyset\right.$, and $\left.\bar{W}=W\right\}$.

Transition function $t_{\mathcal{L}}: \mathcal{L} \rightarrow(1+\mathcal{L})^{A}$ is defined using the input derivatives. Recall that for any partial language $L=\left(L^{1}, L^{2}\right) \in \mathcal{L}, L_{a}=\left(L_{a}^{1}, L_{a}^{2}\right)$, where $L_{a}^{i}=\left\{w \in A^{*} \mid a w \in L^{i}\right\}, \quad i=1,2$. If $a \notin L^{2}$ then $L_{a}$ is undefined. Given any $L=$ $\left(L^{1}, L^{2}\right) \in \mathcal{L}$, the partial automaton structure of $\mathcal{L}$ is given by:

$$
o_{\mathcal{L}}(L)= \begin{cases}1 & \text { if } \varepsilon \in L^{1} \\ 0 & \text { if } \varepsilon \notin L^{1}\end{cases}
$$

and

$$
t_{\mathcal{L}}(L)(a)=\left\{\begin{array}{ll}
L_{a} & \text { if } L_{a} \text { is defined } \\
\emptyset & \text { otherwise }
\end{array} .\right.
$$

Notice that if $L_{a}$ is defined, then $L_{a}^{1} \subseteq L_{a}^{2}, L_{a}^{2} \neq$ $\emptyset$, and $L_{a}^{2}$ is prefix-closed. The following notational conventions will be used: $L \downarrow$ iff $\varepsilon \in L^{1}$ and $L \stackrel{w}{\rightarrow} L_{w}$ iff $L_{w}$ is defined iff $w \in L^{2}$.

Recall from that $\mathcal{L}=\left(\mathcal{L},\left\langle o_{\mathcal{L}}, t_{\mathcal{L}}\right\rangle\right)$ is final among all partial automata: for any partial automaton $S=(S,\langle o, t\rangle)$ there exists a unique homomorphism $l: S \rightarrow \mathcal{L}$. Recall that the unique homomorphism $l$ given by finality of $\mathcal{L}$ maps a state $s \in S$ to the partial language $l(s)=\left(L_{s}^{1}, L_{s}^{2}\right)=$ $\left(\left\{w \in A^{*} \mid s \stackrel{w}{\longrightarrow}\right.\right.$ and $\left.\left.s_{w} \downarrow\right\},\left\{w \in A^{*} \mid s \stackrel{w}{\longrightarrow}\right\}\right)$.

Denote the minimal representation of a partial language $L$ by $\langle L\rangle$, i.e. $\langle L\rangle=\left(D L,\left\langle o_{\langle L\rangle}, t_{\langle L\rangle}\right\rangle\right)$ is a subautomaton of $\mathcal{L}$ generated by $L$. This means that $o_{\langle L\rangle}$ and $t_{\langle L\rangle}$ are uniquely determined by the corresponding structure of $\mathcal{L}$. The carrier set of this minimal representation of $L$ is denoted by $D L$, where $D L=\left\{L_{u} \mid u \in L^{2}\right\}$. Let us call this set the set of derivatives of $L$. Inclusion of partial languages that corresponds to a simulation relation is always meant componentwise.

\section{INTRODUCTION TO DECENTRALIZED SUPERVISORY CONTROL}

Decentralized supervisory control is mostly applied because the system has two or more local controllers each receiving different partial observations of the system. Since communication of the local observations is either not possible or possible but costly, the partial observations of the local controllers differ. Decentralized supervisory control consists in considering local controllers $S_{1}, \ldots, S_{n}$ and breaking the set of controllable and observable events into locally controllable and locally observable events, denoted by $A_{c, i}$, and $A_{o, i}, i=1, \ldots, n$ respectively. The natural projections to locally observable events are denoted by $P_{i}: A^{*} \rightarrow A_{o, i}^{*}$. The action of $P_{i}$ is simply to delete events that are not observable by $S_{i}$.

The following notation will be used: $\{1, \ldots, n\}=$ $Z_{n}$, for any $a \in A: Z_{c}^{a}=\left\{i \in Z_{n}: a \in A_{c . i}\right\}$ and similarly $Z_{o}^{a}=\left\{i \in Z_{n}: a \in A_{o . i}\right\}$. Furthermore, we denote $A_{c}=\cup_{i \in Z_{n}} A_{c, i}, A_{o}=$ $\cup_{i \in Z_{n}} A_{o, i}, A_{u c}=\cap_{i \in Z_{n}}\left(A \backslash A_{c, i}\right)$, and finally $A_{\text {uo }}=\bigcap_{i \in Z_{n}}\left(A \backslash A_{o, i}\right)$.

In the following definition we introduce the notion of of weak derivative (transition). Roughly speaking it disregards locally unobservable events.

Definition 3.1. For an event $a \in A$ define $L \stackrel{P_{i}(a)}{\Rightarrow}$ if $\exists s \in A^{*}: P_{i}(s)=P_{i}(a)$ and $L \stackrel{s}{\rightarrow} L_{s}$. Denote in this case $L \stackrel{P_{i}(a)}{\Rightarrow} L_{s}$. 
Remark 1. Let us introduce the notation for locally unobservable events $L \xi_{i}$ as an abbreviation for $\exists \tau \in A^{*}$ such that $P_{i}(\tau)=\varepsilon$ and $L \stackrel{\tau}{\rightarrow}$. We admit $\tau=\varepsilon$, hence $L \stackrel{\xi}{\Rightarrow}$ is always true. For $a \in A_{o, i}$ our notation means that there exist $\tau, \tau^{\prime} \in\left(A \backslash A_{o, i}\right)^{*}$ such that $L \stackrel{\tau a \tau^{\prime}}{\rightarrow} L_{\tau a \tau^{\prime}}$. This definition can be extended to strings (words in $\left.A^{*}\right)$ in the following way:

$L \stackrel{P_{i}(s)}{\Rightarrow}$ iff $\exists t \in A^{*} \quad: \quad P_{i}(s)=P_{i}(t)$ and $L \stackrel{t}{\rightarrow}$. Denote in this case $L \stackrel{P_{i}(s)}{\Rightarrow} L_{t}$.

\subsection{Coobservability}

There are two control architectures for the decentralized supervisory control. The original control architecture is called C\&P (conjuctive and permissive.) The local supervisor $S_{i}$ is then represented as a mapping $\gamma_{C \& P}\left(S_{i},.\right): P_{i}(L(G)) \rightarrow$ $\Gamma_{i}$, where $\Gamma_{i}=\left\{C \subseteq A: C \supseteq\left(A \backslash A_{c, i}\right)\right\}$ is the set of local control patterns and $\gamma_{C \& P}\left(S_{i}, s\right)$ represents the set of locally enabled events after $S_{i}$ has observed string $s \in A_{o, i}^{*}$. The associated control law of the local supervisor $S_{i}$ is

$$
\begin{gathered}
\gamma_{C \& P}\left(S_{i}, s\right)=\left(A \backslash A_{c, i}\right) \cup \\
\left\{a \in A_{c, i}: \exists s^{\prime} \in K^{2} \cap P_{i}^{-1} P_{i}(s) \text { and } s^{\prime} a \in K^{2}\right\} .
\end{gathered}
$$

The control law of the conjunction of local supervisors $S_{i}, i=1, \ldots, n$ is given by :

$$
\left(\bigwedge_{i} \gamma_{C \& P} S_{i}\right)(w)=\cap_{i=1}^{n} \gamma_{C \& P}\left(S_{i}, P_{i}(w)\right), w \in A^{*}
$$

The necessary and sufficient conditions for a given language to be achieved by a joint action of local supervisors are controllability, $L_{m}(G)$-closedness, and coobservability. The definition of coobservability from Rudie and Willems (1992) can be extended to $\mathrm{n}$ supervisors.

Definition 3.2. (Coobservability.) $K \subseteq L$ is called co-observable with respect to $L$ and $A_{o, i}, i=$ $1, \ldots, n$ if $\left(\forall s \in K^{2}\right),\left(\forall a \in A_{c}: s a \in L^{2}\right)(\exists i \in$ $\left.Z_{c}^{a}\right)$ such that the following implication holds true:

$$
\begin{gathered}
\left(s^{\prime} \in K^{2} \text { and } P_{i}(s)=P_{i}\left(s^{\prime}\right) \text { and } s^{\prime} a \in K^{2}\right) \Rightarrow \\
s a \in K^{2} .
\end{gathered}
$$

Note that the definition of coobservability has been originally formulated for two local supervisors in Rudie and Wonham (1992). This notion of coobservability is needed for the existence of local supervisors that jointly achieve a given language.

The control policy of local supervisors associated to $\mathrm{C} \& \mathrm{P}$ architecture is called permissive, since the default action is to enable an event whenever a local supervisor has an ambiguity what to do with this event. It should be clear that with the permissive local policy we always achieve all strings in the specification language $K$, i.e. $K$ is always contained in the language of the closedloop system. The only concern is the safety, which is expressed by the following definition of $\mathrm{C} \& \mathrm{P}$ coobservability, which states that there always exists a local supervisor that is sure to disable an event resulting in an illegal string. Thus, the following definition of C\&P coobservability - Yoo and Lafortune (2002) is much more intuitive then the original definition of coobservability Rudie and Wonham (1992).

Definition 3.3. ( $C \& P$ coobservability.) $K \subseteq L$ is said to be $C \& P$ co-observable with respect to $L$ and $A_{o, i}, i=1, \ldots, n$ if for all $s \in K^{2}, a \in A_{c}$ such that $s a \in L^{2} \backslash K^{2}$

$$
\exists i \in Z_{c}^{a} \text { with }\left(P_{i}^{-1}\left(P_{i}(s)\right) a \cap K^{2}=\emptyset .\right.
$$

It has been shown in Barrett and Lafortune (2000) that C\&P-coobservability coincides for two supervisors with the 'classical' definition of coobservability introduced in Rudie and Wonham (1992). We will show that the definition of coobservability above (definition 3.2) is equivalent to C\&Pcoobservability and can thus be considered as an extension of the definition given in Rudie and Wonham (1992) to an arbitrary number of local supervisors.

Lemma 2. Coobservability is equivalent to $C \& P$ coobservability.

\section{DECENTRALIZED SUPERVISORY CONTROL AND COALGEBRA}

We have presented in Komenda (2002) a coalgebraic approach to the supervisory control of discrete-event systems with partial observations. It is possible to formulate the basic concepts of decentralized supervisory control using coalgebra. First observe that the concept of observational indistinguishability relation can be easily extended to the family of observation indistinguishability relations associated to local observers. For partial automaton $S$ with initial state $s_{o}$ we define:

Definition 4.1. (Observational indistinguishability relation on $S$.) A binary relation $\operatorname{Aux}_{i}(S), i \in$ $Z_{n}$ on $S$, called an observational indistinguishability relation, is the smallest relation such that:

(i) $\left\langle s_{0}, s_{0}\right\rangle \in A u x_{i}(S)$

(ii) If $\langle s, t\rangle \in A u x_{i}(S)$ then : $\left(s \stackrel{\varepsilon}{\Rightarrow} s_{i}\right.$ for some $s^{\prime} \in S$ and $t \stackrel{\varepsilon}{\xi}_{i} t^{\prime}$ for some $\left.t^{\prime} \in S\right) \Rightarrow$ $\left\langle s^{\prime}, t^{\prime}\right\rangle \in \operatorname{Aux}_{i}(S)$ 
(iii) If $\langle s, t\rangle \in A u x(S)$ then $\forall a \in A_{o, i}:(s \stackrel{a}{\rightarrow}$ $s_{a}$ and $\left.\left.t \stackrel{a}{\rightarrow} t_{a}\right) \Rightarrow\left\langle s_{a}, t_{a}\right)\right\rangle \in \operatorname{Aux}_{i}(S)$.

Since we need to work with the final automaton of partial languages, and for $K \subseteq L$, it is not in general true then $\langle K\rangle$ is a subautomaton of $\langle L\rangle$, we need also the following concept.

Definition 4.2. A binary relation $\operatorname{Aux}_{i}(K, L) \subseteq$ $(D K \times D L)^{2}, i \in Z_{n}$, called an observational indistinguishability relation, is the smallest relation such that:

(i) $\langle(K, L),(K, L)\rangle \in A u x_{i}(K, L)$

(ii) If $\langle(M, N),(Q, R)\rangle \in A u x_{i}(K, L)$ then : $\left((M, N) \stackrel{\xi}{\xi}_{i}\left(M^{\prime}, N^{\prime}\right)\right.$ for some $\left(M^{\prime}, N^{\prime}\right) \in$ $D K \times D L$ and $(Q, R) \stackrel{\xi_{i}}{\Rightarrow}\left(Q^{\prime}, R^{\prime}\right)$ for some $\left.\left(Q^{\prime}, R^{\prime}\right) \in D K \times D L\right) \Rightarrow\left\langle\left(M^{\prime}, N^{\prime}\right),\left(Q^{\prime}, R^{\prime}\right)\right\rangle \in$ $\operatorname{Aux}_{i}(K, L)$

(iii) If $\langle(M, N),(Q, R)\rangle \in A u x_{i}(K, L)$ then $\forall a \in$ $A_{o, i}: \quad\left((M, N) \stackrel{a}{\rightarrow}\left(M_{a}, N_{a}\right)\right.$ and $(Q, R) \stackrel{a}{\rightarrow}$ $\left.\left(Q_{a}, R_{a}\right)\right) \Rightarrow\left\langle\left(M_{a}, N_{a}\right),\left(Q_{a}, R_{a}\right)\right\rangle \in \operatorname{Aux}_{i}(K, L)$

For $\langle(M, N),(Q, R)\rangle \in D K \times D L$ we write $(M, N) \approx_{\operatorname{Aux}(i)}^{K, L}(Q, R)$ whenever

$\langle(M, N),(Q, R)\rangle \in \operatorname{Aux}_{i}(K, L)$. Similarly as for the centralized $\operatorname{Aux}(K)$ we have:

Lemma 3. For given partial languages $K, L$ :

$\langle(M, N),(Q, R)\rangle \in \operatorname{Aux}_{i}(K, L)$ iff there exist two strings $s, s^{\prime} \in K^{2}$ such that $P_{i}(s)=P_{i}\left(s^{\prime}\right)$ and $M=K_{s}, N=L_{s}, Q=K_{s^{\prime}}$, and $R=L_{s^{\prime}}$.

Now the C\&P coobservability can be formulated within the coalgebraic framework of partial automata.

Definition 4.3. (C\&P Coobservability relation.) Given two (partial) languages $\mathrm{K}$ and $\mathrm{L}$, a binary relation $C O(K, L) \subseteq D K \times D L$ is called a $C \& P$ coobservability relation if for any $\langle M, N\rangle \in$ $C O(K, L)$ the following items hold:

(i) $\forall a \in A: M \stackrel{a}{\rightarrow} \Rightarrow N \stackrel{a}{\rightarrow}$ and $\left\langle M_{a}, N_{a}\right\rangle \in$ $C O(K, L)$

(ii) $\forall a \in A_{c}: N \stackrel{a}{\rightarrow} \Rightarrow\left[\left(\exists i \in Z_{c}^{a}\right)\right.$ such that $\left(M^{\prime} \in D K, N^{\prime} \in D L:\left(M^{\prime}, N^{\prime}\right) \approx_{A u x(i)}^{K, L}(M, N)\right.$ and $\left.M^{\prime} \stackrel{a}{\rightarrow}\right) \Rightarrow M \stackrel{a}{\rightarrow}$ ].

For $M \in D K$ and $N \in D L$ we write $M \approx_{C O(K, L)}$ $N$ whenever there exists a C\&P coobservability relation $C O(K, L)$ on $D K \times D L$ such that $\langle M, N\rangle \in C O(K, L)$. In order to check whether for a given pair of (partial) languages ( $K$ and $L$ ), $K$ is C\&P coobservable with respect to $L$ and
$A_{o, i}, i=1, \ldots, n$, it is sufficient to establish a C\&P coobservability relation $O(K, L)$ on $D K \times$ $D L$ such that $\langle K, L\rangle \in O(K, L)$. Indeed, we have:

Theorem 4. A (partial) language $K$ is $C \& P$ coobservable with respect to $L$ (where $K \subseteq L$ ) and $A_{o, i}, i=1, \ldots, n$ iff $K \approx_{C O(K, L)} L$.

PROOF. $(\Rightarrow)$ Let $\mathrm{K}$ be $\mathrm{C} \& \mathrm{P}$ coobservable with respect to $\mathrm{L}$. Denote

$$
C O(K, L)=\left\{\left\langle K_{u}, L_{u}\right\rangle \in D K \times D L \mid u \in K^{2}\right\} .
$$

Let us show that $C O(K, L)$ is a $\mathrm{C} \& \mathrm{P}$ coobservability relation.

Let $\langle M, N\rangle \in C O(K, L)$. We can assume that $M=K_{s}$ and $N=L_{s}$ for $s \in K^{2}$. We must show that conditions (i) and (ii) are safisfied.

(i) Let $M \stackrel{a}{\rightarrow}$ for $a \in A$. Notice that $K \subseteq L$ implies that for any $u \in K^{2}, K_{u} \subseteq L_{u}$. In particular $N \stackrel{a}{\rightarrow}$, because $M=K_{s} \subseteq L_{s}=N$ and it follows from the definition of $C O(K, L)$ that $\left\langle M_{a}, N_{a}\right\rangle \in C O(K, L)$.

(ii) Let $N \stackrel{a}{\rightarrow}$ for $a \in A_{c}$. Then we have $s a \in L^{2}$ and recall that $s \in K^{2}$. Then by C\&P coobservability of $K$ with respect to $L$ there exists $i \in Z_{c}^{a}$ such that whenever there is a string $s^{\prime} a \in K^{2}$ with $P_{i}\left(s^{\prime}\right)=P_{i}(s)$, then also $s a \in K^{2}$. Using Lemma 3 this means that there exists $i \in Z_{c}^{a}$ such that whenever $\left(M^{\prime}, N^{\prime}\right) \approx_{A u x(i)}^{K, L}(M, N): M^{\prime} \stackrel{a}{\rightarrow}$, then $M \stackrel{a}{\rightarrow}$. Indeed this means that there exist $s^{\prime}, s^{\prime \prime} \in A^{*}: M^{\prime}=K_{s^{\prime}}, N^{\prime}=L_{s^{\prime}}, M=K_{s^{\prime \prime}}=K_{s}$, $N=L_{s^{\prime \prime}}=L_{s}$, and $P_{i}\left(s^{\prime \prime}\right)=P_{i}\left(s^{\prime}\right)$. Note that it can be that $s=s^{\prime \prime}$. We have $s^{\prime \prime} \in K^{2}$ and $s^{\prime \prime} a \in L^{2}$. By applying the coobservability of $K$, where $s^{\prime \prime}$ plays the role of $s$, it follows that $s^{\prime \prime} a \in K$, i.e. $K_{s^{\prime \prime}}=M \stackrel{a}{\rightarrow}$. Hence $C O(K, L)$ is a $\mathrm{C} \& \mathrm{P}$ coobservability relation.

$(\Leftarrow)$ Let $K \approx_{C O(K, L)} L$. Let us show that $K$ is C\&P coobservable with respect to $L$. For this purpose, let $s \in K^{-2}$ and $a \in A_{c}$ such that $s a \in L^{2}$. Then $s \in K^{2} \cap L^{2}$, i.e. $L \stackrel{s}{\rightarrow}$ and $K \stackrel{s}{\rightarrow}$, whence from (i) of definition 4.3 inductively applied $K_{s} \approx C O(K, L) \quad L_{s}$. Now, sa $\in L^{2}$ means that $L_{s} \stackrel{a}{\rightarrow}$, hence by definition of 4.3 there exists $i \in Z_{c}^{a}$ such that whenever $\left(M^{\prime}, N^{\prime}\right) \approx_{\operatorname{Aux}(i)}^{K, L}$ $\left(K_{s}, L_{s}\right): \quad M^{\prime} \stackrel{a}{\rightarrow}$, then $K_{s} \stackrel{a}{\rightarrow}$. According to Lemma 3 we have for $P_{i}\left(s^{\prime}\right)=P_{i}(s)$ that $\left(K_{s^{\prime}}, L_{s^{\prime}}\right) \approx_{\operatorname{Aux}(i)}^{K, L}\left(K_{s}, L_{s}\right)$. Also notice that $s^{\prime} a \in$ $K^{2}$ is equivalent to $K_{s^{\prime}} \stackrel{a}{\rightarrow}$. But this means that there exists $i \in Z_{c}^{a}$ such that whenever there is a string $s^{\prime} a \in K^{2}$ with $P_{i}\left(s^{\prime}\right)=P_{i}(s)$, then also $s a \in K^{2}$, i.e. $K$ is $\mathrm{C} \& \mathrm{P}$ coobservable with respect to $L$ and $A_{o, i}, i=1, \ldots, n$. 


\section{DECOMPOSABILITY AND STRONG DECOMPOSABILITY}

It is known that coobservability is not preserved under unions. Therefore the existence of supremal coobservable sublanguages cannot be guaranted. For this reason, stronger notions of decomposable and strongly decomposable languages have been studied.

Definition 5.1. (Decomposability.) Let $K \subseteq L$ be given partial languages. $K$ is said to be decomposable with respect to $L$ and $A_{o, i}, i=1, \ldots, n$ if $K^{2}=L^{2} \cap \bigcap_{i=1}^{n} P_{i}^{-1}\left(P_{i}\left(K^{2}\right)\right)$.

Let us introduce now a binary relation that corresponds to the decomposability.

Definition 5.2. (Decomposability relation.) Given two languages $K, L$ with $K \subseteq L$, a binary relation $D(K, L)$ on $D K \times D L$ is said to be a decomposability relation if for any $\langle M, N\rangle \in D(K, L)$ the following items hold:

(i) $\forall a \in A: M \stackrel{a}{\rightarrow} M_{a} \Rightarrow N \stackrel{a}{\rightarrow} N_{a}$ and $\left\langle M_{a}, N_{a}\right\rangle \in$ $D(K, L)$

(ii) $\forall u \in A_{u o}: N \stackrel{u}{\rightarrow} \Rightarrow M \stackrel{u}{\rightarrow}$

(iii) $\forall a \in A_{o}: N \stackrel{a}{\rightarrow}$ and $\left[\forall i \in Z_{n}: \exists M^{(i)} \in\right.$ $D K$ and $N^{(i)} \in D L:\left(M^{(i)}, N^{(i)}\right) \quad \approx_{A u x(i)}^{K, L}$ $(M, N)$ and $\left.\left.M^{(i)} \stackrel{a}{\rightarrow}\right)\right] \Rightarrow M \stackrel{a}{\rightarrow}$.

Using strong and weak transitions, we obtain:

Lemma $5 . K$ is decomposable with respect to $L$ and $A_{o, i}, i=1, \ldots, n$ iff $\forall w \in A^{*}$ :

$\left\{L \stackrel{w}{\rightarrow}\right.$ and $\left.\left(\forall i=1, \ldots, n: K \stackrel{P_{i}(w)}{\Rightarrow}\right)\right\} \Rightarrow K \stackrel{w}{\rightarrow}$.

Theorem 6. A (partial) language $K$ is decomposable with respect to $L$ and $A_{o, i}, i=1, \ldots, n$ (with $K \subseteq L)$ iff there exists a decomposability relation $D(K, L) \subseteq D K \times D L$ such that $\langle K, L\rangle \in D(K, L)$.

PROOF. $(\Rightarrow)$ Let $K$ be decomposable with respect to $L$ and $A_{o, i}, i=1, \ldots, n$. Denote

$$
R=\left\{\left\langle K_{u}, L_{u}\right\rangle \mid u \in K^{2}\right\} \subseteq D K \times D L .
$$

Let us show that $R$ is indeed a decomposability relation. Assume that $\langle M, N\rangle \in R$. We can assume that $M=K_{s}$ and $N=L_{s}$ for $s \in K^{2}$. We must show that conditions (i)-(iii) of decomposability relations are satisfied.

(i) If for $a \in A$ we have $M \stackrel{a}{\rightarrow}$ (i.e. $a \in M^{2}$ ) then $a \in N^{2}$, i.e. $N \stackrel{a}{\rightarrow}$, because $K \subseteq L$ implies that $M=K_{s} \subseteq L_{s}=N$. Moreover, it follows from the definition of $R$ that $\left\langle M_{a}, N_{a}\right\rangle \in R$.

(ii) Let $u \in A_{u o}$ such that $N \stackrel{u}{\rightarrow}$. Then $L \stackrel{s u}{\rightarrow}$, and since $K \stackrel{s}{\rightarrow}$ and $\forall i=1, \ldots, n: \quad P_{i}(s u)=P_{i}(s)$, trivially $\forall i=1, \ldots, n$ we have $K \stackrel{P_{i}(s u)}{\Rightarrow}$. Therefore by Lemma $5 K \stackrel{s u}{\rightarrow}$, i.e. $M \stackrel{u}{\rightarrow}$.

(iii) If for $a \in A_{o}: N \stackrel{a}{\rightarrow}$ and $[\forall i \in\{1, \ldots, n\}$ : $\exists M^{(i)} \in D K$ and $N^{(i)} \in D L:\left(M^{(i)}, N^{(i)}\right) \approx_{A u x(i)}^{K, L}$

$(M, N)$ and $\left.\left.M^{(i)} \stackrel{a}{\rightarrow}\right)\right]$ then $L \stackrel{s a}{\rightarrow}$ and $\forall i=$ $1, \ldots, n \exists s_{i}, s_{i}^{\prime} \in K^{2}$ such that $K_{s}=K_{s_{i}}$, $L_{s}=L_{s_{i}}, M^{i}=K_{s_{i}^{\prime}}, N^{i}=L_{s_{i}^{\prime}}$, where $P_{i}\left(s_{i}\right)=$ $P_{i}\left(s_{i}^{\prime}\right)$ and $K_{s_{i}^{\prime}} \stackrel{a}{\rightarrow}$, hence $K \stackrel{P_{i}\left(s_{i} a\right)}{\Rightarrow}$. Recall that $L_{s_{i}}=L_{s} \stackrel{s a}{\rightarrow}$. By Lemma 5 we obtain $K \stackrel{s_{i} a}{\rightarrow}$, i.e. $M=K_{s_{i}} \stackrel{a}{\rightarrow}$.

$(\Leftarrow)$ Let there exists a decomposability relation $D(K, L)$ such that $\langle K, L\rangle \in D(K, L)$. We show by induction on the structure of the string $w \in A^{*}$ that the implication of Lemma 5 holds true. For $w=\varepsilon$ it is trivially true, because $K^{2}$ is closed (i.e. $K \stackrel{\varepsilon}{\rightarrow}$ ). Suppose now that for $w \in A^{*}: L \stackrel{w}{\rightarrow}$ and $\left(K \stackrel{P_{i}(w)}{\Rightarrow} \forall i=1, \ldots, n\right)$ imply that $K \stackrel{w}{\rightarrow}$.

Let $L \stackrel{w a}{\rightarrow}$ and $K \stackrel{P_{i}(w a)}{\Rightarrow} \forall i=1, \ldots, n$. This implies in particular that $L \stackrel{w}{\rightarrow}$ and $K \stackrel{P_{i}(w)}{\Rightarrow} \forall i=1, \ldots, n$, hence by the induction hypothesis $K \stackrel{w}{\rightarrow}$. Since $\langle K, L\rangle \in D(K, L)$, by inductive application of (i) of the definition of decomposability relation we obtain that $\left\langle K_{w}, L_{w}\right\rangle \in D(K, L)$. Now suppose first $a \in A_{u o}$ and $L_{w} \stackrel{a}{\rightarrow}$, then we obtain by (ii) of the definition of decomposability relation $K_{w} \stackrel{a}{\rightarrow}$, i.e. $K \stackrel{w a}{\rightarrow}$. If $a \in A_{o}$, then $\forall i: K \stackrel{P_{i}(w a)}{\Rightarrow}$ means that for all $i=1, \ldots, n$ there exist $s_{i} \in$ $A^{*}:\left(K_{s_{i}}, L_{s_{i}}\right) \approx_{A u x(i)}^{K, L}\left(K_{w}, L_{w}\right)$ and $K_{s_{i}} \stackrel{a}{\rightarrow}$. By application of (iii) of decomposability relation we have $K_{w} \stackrel{a}{\rightarrow}$, i.e. $K \stackrel{w a}{\rightarrow}$.

Decomposability is related to $C \& P$-coobservability. The following theorem holds.

Theorem 7. Let $A_{c} \subseteq A_{o}$, for $i \in Z_{n}: A_{o, i} \cap$ $A_{c} \subseteq A_{c, i}$, and $K \subseteq L$ be decomposable wrt $L$ and $A_{o, i}, i=1, \ldots, n$. Then $K$ is C\&P-coobservable wrt $L$ and $A_{o, i}, i \in Z_{n}$.

PROOF. Let $K$ be decomposable wrt $L$ and $A_{o, i}, i=1, \ldots, n$. We show that if

$$
R=\left\{\left\langle K_{u}, L_{u}\right\rangle \mid u \in K^{2}\right\}
$$

is a decomposability relation, it is also a C\&Pcoobservability relation. Take $M=K_{s}$ and $N=$ $L_{s}$ for a $s \in K^{2}$. Then (i) of coobservability relations trivially holds. Assume by contradiction that (ii) does not hold, i.e. there exists $a \in A_{c}$ such that $N \stackrel{a}{\rightarrow}$ and $\left(\forall i \in Z_{n}: a \in A_{c, i}\right)$ $\exists\left(M^{(i)}, N^{(i)}\right) \approx_{A u x(i)}^{K, L}(M, N)$ such that $M^{(i)} \stackrel{a}{\rightarrow}$, while $M \stackrel{q}{\rightarrow}$. Recall that $K \stackrel{s}{\rightarrow} M$. The last condition means that $\left(\forall i \in Z_{n}: a \in A_{c, i}\right)$ : $K \stackrel{P_{i}(s a)}{\Rightarrow}$, because by Lemma 3 there exist $s_{i}, s_{i}^{\prime} \in$ $K^{2}$ not all necessarily different such that $M=$ $K_{s_{i}}, N=L_{s_{i}}, M^{i}=K_{s_{i}^{\prime}}, N^{i}=L_{s_{i}^{\prime}}$, with 
$P_{i}\left(s^{i}\right)=P_{i}(s)$ and $K_{s^{i}} \stackrel{a}{\rightarrow}$. Now, if $a \notin A_{c, i}$, then $a \notin A_{c} \cap A_{o, i}$. Since $a \in A_{c}$, there must be $a \notin A_{o, i}$. This means that $K \stackrel{s_{i}}{\rightarrow} M$ and $K \stackrel{P_{i}\left(s_{i} a\right)}{\rightarrow}$ (because $\left.M^{(i)} \stackrel{a}{\rightarrow}\right)$. Furthermore, $P_{i}(s a)=P_{i}(a)$ for $i \in$ $Z_{n} a \notin A_{o, i}$. We conclude that for $a \in A_{c} \subseteq A_{o}$ we have $L \stackrel{s_{i} a}{\rightarrow}$ and $\forall i \in Z_{n}: K \stackrel{P_{i}\left(s_{i} a\right)}{\Rightarrow}$, i.e. from decomposability of $K$ we have $M=K_{s_{i}} \stackrel{a}{\rightarrow}$. This is a contradiction. Therefore, we conclude that $K$ is $C \& P$-coobservable.

Theorem 8. Let $A_{c} \subseteq A_{o}, K \subseteq L$ is controllable, and $C \& P$-coobservable wrt $L$ and $A_{o, i}, i \in Z_{n}$. Then $K$ is decomposable wrt $L$ and $A_{o, i}, i \in Z_{n}$.

PROOF. Let $K$ be C\&P-coobservable wrt $L$ and $A_{o, i}, i \in Z_{n}$. We show that

$$
R=\left\{\left\langle K_{u}, L_{u}\right\rangle \mid u \in K^{2}\right\}
$$

is a decomposability relation. Take $M=K_{s}$ and $N=L_{s}$ for $s \in K^{2}$. First of all, (i) of decomposability relations is trivial. Also (ii) easily follows from controllability of $K$ with respect to $L$ and $A_{u c}$. Indeed, it is sufficient to notice that $A_{u o}=A \backslash A_{o} \subseteq A \backslash A_{c}=A_{u c}$. Then (ii) follows from controllability of $K$. Assume by contradiction that (iii) does not hold, i.e. there exists $a \in A_{o}$ such that $N \stackrel{a}{\rightarrow}$ and $\forall i \in Z_{n} \exists\left(M^{(i)}, N^{(i)}\right) \approx_{\operatorname{Aux}(i)}^{K, L}(M, N)$ such that $M^{(i)} \stackrel{a}{\rightarrow}$, while $M \stackrel{a}{\rightarrow}$. We have also $a \in A_{c}$, because otherwise by controllability we would have $M \stackrel{a}{\rightarrow}$. This means there exists at least one $j \in Z_{n}$ such that $a \in A_{c, j}$. Since the above condition holds for all $i \in Z_{n}$, in particular it holds for all $i \in Z_{n}$ such that $a \in A_{c, i}$. But this is a contradiction with $C \& P$-coobservability. Therefore, we conclude that $K$ is decomposable.

The set of decomposable sublanguages is not closed under unions either. There is yet a stronger condition, called strong decomposability, which is preserved by arbitrary unions.

Definition 5.3. (Strong Decomposability.) Let $K \subseteq L$ be given partial languages. $K$ is said to be strongly decomposable with respect to $L$ and $A_{o, i}, i=1, \ldots, n$ if $K^{2}=L^{2} \cap \cup_{i=1}^{n} P_{i}^{-1}\left(P_{i}\left(K^{2}\right)\right)$.

Now we introduce a binary relation that corresponds to strong decomposability.

Definition 5.4. (Strong decomposability relation.) Given two languages $K, L$ with $K \subseteq L$, a binary relation $S D(K, L)$ on $D K \times D L$ is said to be a strong decomposability relation if for any $\langle M, N\rangle \in$ $S D(K, L)$ the following items hold:

(i) $\forall a \in A: M \stackrel{a}{\rightarrow} M_{a} \Rightarrow N \stackrel{a}{\rightarrow} N_{a}$ and $\left\langle M_{a}, N_{a}\right\rangle \in$ $D(K, L)$ (ii) $\forall u \in \cup_{i=1}^{n}\left(A \backslash A_{o, i}\right): N \stackrel{u}{\rightarrow} \Rightarrow M \stackrel{u}{\rightarrow}$

(iii) $\forall a \in \cap_{i=1}^{n} A_{o, i}: \quad N \stackrel{a}{\rightarrow}$ and $[\exists i \in$ $Z_{n}$ and $\left(\exists M^{\prime} \in D K, N^{\prime} \in D L\right.$ : $\left(M^{\prime}, N^{\prime}\right) \approx_{A u x(i)}^{K, L}(M, N)$ and $\left.\left.M^{\prime} \stackrel{a}{\rightarrow}\right)\right] \Rightarrow$ $M \stackrel{a}{\rightarrow}$.

We have the following theorem:

Theorem 9. A (partial) language $K$ is strongly decomposable with respect to $L$ and $A_{o, i}, i=$ $1, \ldots, n$ (with $K \subseteq L$ ) iff there exists a strong decomposability relation $S D(K, L) \subseteq D K \times D L$ such that $\langle K, L\rangle \in S D(K, L)$.

\section{CONCLUSION}

Decentralized supervisory control of DES has been treated by coalgebraic techniques. Coobservability, decomposability, and strong decomposability have been characterized by appropriate relations in this framework. Exact relationships between properties like $\mathrm{C} \& \mathrm{P}$ coobservability and decomposability have been derived using these relational characterizations.

\section{REFERENCES}

G.Barrett and S. Lafortune. Decentralized supervisory control with communicating controllers. IEEE Trans. on Automatic Control, 45, p. 1620$1638,2000$.

S.G. Cassandras and S. Lafortune. Introduction to Discrete Event Systems. Kluwer Academic Publishers, Dordrecht 1999.

J. Komenda. Coalgebra and coinduction in discrete-event control. Submitted to Siam Journal on Control and Optimization, October 2002.

S. Lafortune and E. Chen. The infimal closed and controllable superlanguage and its applications in supervisory control. IEEE Trans. on Automatic Control, Vol. 35,N 4, p. 398-405, 1990.

A. Overkamp and J.H. van Schuppen. Maximal solutions in decentralized supervisory control, SIAM Journal on Control and Optimization, Vol. 39, No.2, pp. 492-511, 2000.

J.J.M.M. Rutten. Coalgebra, Concurrency, and Control. Research Report CWI, SEN3, Amsterdam, 1999.

K. Rudie and W.M. Wonham. Think Globally, Act Locally: Decentralized Supervisory Control. IEEE Trans. on Automatic Control, Vol. 37,N 11, p. 1692-1708, 1992.

T.S. Yoo and S. Lafortune. General Architecture for Decentralized Supervisory Contol of Discrete-Event Systems. Discrete Event Dynamic Systems: Theory and Applications, 12, 335-377, 2002. 